Supplement of Atmos. Chem. Phys., 20, 12795-12811, 2020

https://doi.org/10.5194/acp-20-12795-2020-supplement

(C) Author(s) 2020. This work is distributed under

the Creative Commons Attribution 4.0 License.

(c) (1)
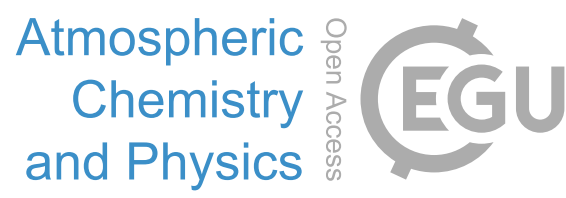

Supplement of

\title{
Improved inversion of aerosol components in the atmospheric column from remote sensing data
}

\section{Ying Zhang et al.}

Correspondence to: Zhengqiang Li (lizq@radi.ac.cn)

The copyright of individual parts of the supplement might differ from the CC BY 4.0 License. 


\section{S1. Estimation of CRIs for the fine and coarse modes}

In order to separate the complex refractive index (CRI) for different modes, first the volume size distribution (VSD) needs to be separated into complete log-normal functions following the VSD breakdown method. The multi-modal log-normal distributions fits the AERONET-retrieved VSD by the following formula:

$$
\frac{d V(r)}{d \ln r}=\sum_{i=1, m} \frac{C_{i}}{\sqrt{2 \pi}\left|\ln \sigma_{i}\right|} \exp \left[-\frac{1}{2}\left(\frac{\ln r-\ln r_{i}}{\ln \sigma_{i}}\right)^{2}\right], \mathrm{m}=1,2, \ldots,
$$

where $\mathrm{d} V / \mathrm{d} \ln r$ (in unit of $\mu \mathrm{m}^{3} / \mu \mathrm{m}^{2}$ ) is the volume particle size distribution, $C_{i}\left(\mu \mathrm{m}^{3} / \mu \mathrm{m}^{2}\right), r_{i}(\mu \mathrm{m})$, and $\ln \sigma_{i}$ are the volume modal concentration, median radius, and standard deviation of $\ln r_{i}$ for each $\log$ normal mode, respectively. Based on the separated VSD for the fine and coarse mode, a limitedmemory optimization algorithm is employed to retrieve the CRIs. The real part (n) of sub-CRIs is spectrally independent, and the imaginary part $(\mathrm{k})$ of sub-CRIs varies with wavelength:

$$
\begin{aligned}
n_{f / c}(\lambda) & =n_{f / c} \\
k_{f / c}(\lambda) & =\left\{\begin{array}{c}
k_{f / c, 440} \\
k_{f / c}
\end{array}\right.
\end{aligned}
$$

where $\lambda$ denotes the standard wavelengths of AERONET products and " $f$ " and " $c$ " represent the fine and coarse modes, respectively. The details of the CRIs separating process are presented in Zhang et al. (2017).

In the study of Zhang et al. (2017), the uncertainties in the estimated complex refractive indices of the fine and coarse modes were evaluated using the three typical aerosol models presented in table S1. The typical uncertainties in the retrieved complex refractive indices of fine and coarse modes for these models are listed in the table 2 . The total uncertainty (TU) is calculated by error propagation using the formula:

$$
T U=\sqrt{x_{\Delta \tau}^{2}+x_{\Delta \omega}^{2}+x_{\Delta V S D}^{2}}
$$

where $x$ represents the uncertainty of the sub-CRIs for each aerosol type. The biases of the input parameter (aerosol optical depth $(\tau)$, single scattering albedo $(\omega)$ and VSD) uncertainties are set to 0.01 , -0.03 and $15 \%-35 \%$ in the WS, BB and DU aerosol models, respectively. The uncertainty in the relative humidity is twice the observational error given by the World Meteorological Organization (WMO, 2008).

Table S1. Typical aerosol models (WS: Water-soluble, BB: Biomass burning, DU: Dust) parameters 
and relative humidity.

\begin{tabular}{ccccccccccccc}
\hline Type & $\boldsymbol{r}_{\boldsymbol{1}}$ & $\boldsymbol{r}_{\boldsymbol{2}}$ & $\boldsymbol{\sigma}_{\boldsymbol{1}}$ & $\boldsymbol{\sigma}_{\boldsymbol{2}}$ & $\boldsymbol{C}_{\boldsymbol{1}} / \boldsymbol{C}_{\boldsymbol{2}}$ & $\boldsymbol{n}_{\boldsymbol{f}}$ & $\boldsymbol{k}_{\boldsymbol{f}, \mathbf{4 4 0}}$ & $\boldsymbol{k}_{\boldsymbol{f}}$ & $\boldsymbol{n}_{\boldsymbol{c}}$ & $\boldsymbol{k}_{\boldsymbol{c}, 440}$ & $\boldsymbol{k}_{\boldsymbol{c}}$ & $\boldsymbol{R} \boldsymbol{H}$ \\
\hline WS & 0.118 & 1.17 & 0.6 & 0.6 & 2 & 1.45 & 0.0035 & 0.0035 & 1.53 & 0.008 & 0.008 & $70 \%$ \\
BB & 0.132 & 4.50 & 0.4 & 0.6 & 4 & 1.52 & 0.025 & 0.025 & 1.53 & 0.008 & 0.008 & $55 \%$ \\
DU & 0.100 & 3.40 & 0.6 & 0.8 & 0.066 & 1.53 & 0.008 & 0.008 & 1.53 & 0.008 & 0.008 & $35 \%$ \\
\hline
\end{tabular}

Table S2. Typical uncertainties $( \pm \Delta)$ of the estimated complex refractive indices of the fine and coarse modes and relative humidity.

\begin{tabular}{cccccccc}
\hline Type & $\boldsymbol{\Delta} \boldsymbol{n}_{\boldsymbol{f}}$ & $\boldsymbol{\Delta} \boldsymbol{k}_{\boldsymbol{f}, \boldsymbol{4 4 0}}$ & $\boldsymbol{\Delta} \boldsymbol{k}_{\boldsymbol{f}}$ & $\boldsymbol{\Delta} \boldsymbol{n}_{\boldsymbol{c}}$ & $\boldsymbol{\Delta} \boldsymbol{k}_{\boldsymbol{c}, \boldsymbol{4 4 0}}$ & $\boldsymbol{\Delta} \boldsymbol{k}_{\boldsymbol{c}}$ & $\boldsymbol{\Delta} \boldsymbol{R H}$ \\
\hline WS & 0.0197 & 0.0015 & 0.0004 & 0.0667 & 0.0036 & 0.0029 & $10 \%$ \\
BB & 0.0270 & 0.0039 & 0.0030 & 0.0500 & 0.0016 & 0.0016 & $10 \%$ \\
DU & 0.0780 & 0.0036 & 0.0040 & 0.0447 & 0.0016 & 0.0036 & $10 \%$ \\
\hline
\end{tabular}

S2. The averaged mass fraction of aerosol components

Table S3. The averaged mass fraction of aerosol components at SONET sites shown in figure 7.

\begin{tabular}{|c|c|c|c|c|c|c|c|c|c|c|c|}
\hline \multirow{2}{*}{ Site } & \multicolumn{5}{|c|}{ Fine mode } & \multicolumn{3}{|c|}{ Coarse mode } & \multirow{2}{*}{ AW } & \multirow{2}{*}{ IS } & \multirow{2}{*}{$\mathbf{O M}$} \\
\hline & BC & WIOM & WSOM & $\mathbf{A N}$ & $\mathbf{A W}_{\mathbf{f}}$ & DU & SC & $\mathbf{A W}_{\mathbf{c}}$ & & & \\
\hline Lhasa & $0.43 \%$ & $7.85 \%$ & $6.47 \%$ & $1.89 \%$ & $2.01 \%$ & $81.34 \%$ & $0.00 \%$ & $0.00 \%$ & $2.01 \%$ & $1.89 \%$ & $14.33 \%$ \\
\hline Zhangye & $0.05 \%$ & $2.43 \%$ & $2.36 \%$ & $2.31 \%$ & $1.79 \%$ & $70.23 \%$ & $11.53 \%$ & $9.30 \%$ & $11.09 \%$ & $13.84 \%$ & $4.79 \%$ \\
\hline Kashgar & $0.08 \%$ & $4.57 \%$ & $2.63 \%$ & $2.51 \%$ & $1.19 \%$ & $65.63 \%$ & $17.09 \%$ & $6.30 \%$ & $7.49 \%$ & $19.60 \%$ & $7.20 \%$ \\
\hline Minqin & $0.11 \%$ & $2.97 \%$ & $2.33 \%$ & $6.13 \%$ & $2.33 \%$ & $65.70 \%$ & $12.20 \%$ & $8.23 \%$ & $10.56 \%$ & $18.33 \%$ & $5.30 \%$ \\
\hline Xi'an & $0.60 \%$ & $4.74 \%$ & $9.62 \%$ & $10.08 \%$ & $6.56 \%$ & $60.75 \%$ & $3.37 \%$ & $4.28 \%$ & $10.84 \%$ & $13.45 \%$ & $14.37 \%$ \\
\hline Beijing & $0.69 \%$ & $5.91 \%$ & $7.67 \%$ & $10.65 \%$ & $6.08 \%$ & $62.86 \%$ & $2.85 \%$ & $3.29 \%$ & $9.38 \%$ & $13.49 \%$ & $13.59 \%$ \\
\hline Nanjing & $0.96 \%$ & $6.26 \%$ & $15.13 \%$ & $13.79 \%$ & $9.68 \%$ & $46.94 \%$ & $3.14 \%$ & $4.10 \%$ & $13.78 \%$ & $16.93 \%$ & $21.39 \%$ \\
\hline Shanghai & $1.30 \%$ & $5.14 \%$ & $10.78 \%$ & $17.68 \%$ & $10.33 \%$ & $46.62 \%$ & $3.87 \%$ & $4.28 \%$ & $14.61 \%$ & $21.55 \%$ & $15.92 \%$ \\
\hline Harbin & $0.97 \%$ & $5.43 \%$ & $12.45 \%$ & $15.36 \%$ & $10.80 \%$ & $50.50 \%$ & $2.21 \%$ & $2.26 \%$ & $13.07 \%$ & $17.57 \%$ & $17.88 \%$ \\
\hline Hefei & $0.80 \%$ & $3.33 \%$ & $11.51 \%$ & $14.86 \%$ & $10.34 \%$ & $49.79 \%$ & $3.91 \%$ & $5.46 \%$ & $15.80 \%$ & $18.77 \%$ & $14.84 \%$ \\
\hline Songshan & $0.59 \%$ & $8.14 \%$ & $6.31 \%$ & $12.09 \%$ & $6.63 \%$ & $59.65 \%$ & $3.10 \%$ & $3.50 \%$ & $10.13 \%$ & $15.18 \%$ & $14.45 \%$ \\
\hline Chengdu & $0.58 \%$ & $2.72 \%$ & $11.90 \%$ & $22.54 \%$ & $9.34 \%$ & $42.10 \%$ & $3.65 \%$ & $7.17 \%$ & $16.51 \%$ & $26.19 \%$ & $14.62 \%$ \\
\hline Zhoushan & $0.33 \%$ & $3.55 \%$ & $6.84 \%$ & $17.06 \%$ & $14.86 \%$ & $42.82 \%$ & $5.83 \%$ & $8.70 \%$ & $23.56 \%$ & $22.89 \%$ & $10.40 \%$ \\
\hline Guangzhou & $0.64 \%$ & $2.84 \%$ & $8.18 \%$ & $23.80 \%$ & $18.26 \%$ & $31.54 \%$ & $4.28 \%$ & $10.46 \%$ & $28.72 \%$ & $28.08 \%$ & $11.02 \%$ \\
\hline Haikou & $0.83 \%$ & $2.32 \%$ & $9.89 \%$ & $22.03 \%$ & $14.42 \%$ & $33.29 \%$ & $6.90 \%$ & $10.32 \%$ & $24.74 \%$ & $28.93 \%$ & $12.21 \%$ \\
\hline Sanya & $0.55 \%$ & $0.32 \%$ & $14.27 \%$ & $24.78 \%$ & $9.46 \%$ & $37.41 \%$ & $3.21 \%$ & $10.00 \%$ & $19.45 \%$ & $27.99 \%$ & $14.59 \%$ \\
\hline
\end{tabular}

\section{S3. The comparison of aerosol components}

We have made the comparison of the aerosol components retrieved with the new algorithm presented 
here, with those from Zhang et al (2018). The number of retrievals in this study is less than that in Zhang et al. (2018). There are three reasons: (1) The input data is more rigorously filtered (Li et al., 2017); (2) the residuals are increased using the new algorithm; (3) stricter residual constraints are used. From these, we can obtain more reasonable inversion aerosol components. Figure S1 shows the comparison of aerosol components (OM, BC and $\mathrm{AN})$ in the fine mode in atmospheric column from this study and those from Zhang et al., 2018 with reference $\mathrm{PM}_{1}$ composition data which were measured by a High-Resolution Aerosol Mass Spectrometer at ground level. We use the boundary layer height of lidar (obtained from Zhang et al. 2018) to calculate the concentration of the atmospheric column to the near surface. The results show that OM components from the improved algorithm are not better than from Zhang et al. (2018). Black carbon is closer to the identity line although the correlation coefficient is slightly smaller than in 2018. For AN, a water-soluble inorganic salt, the new algorithm shows a good effect. The slope with ground observations changes from negative to positive. In our opinion, such a comparison is not sufficient due to the various vertical distribution of aerosol components. In future studies, we will make a more detailed and comprehensive comparison.

This comparison does not show the comprehensive advantages of the new algorithm. Although the algorithm in this paper has been improved, the basic assumption (e.g. Nonhygroscopic assumption of OM mixture) is not different from the paper in 2018. The current algorithm can easily add more kinds of hygroscopic components without obtaining the single component hygroscopic formula (A polynomial in water activity and solution concentration in paper of 2018 Eqs (5) \& (6)) to better solve the problem of OM mixture.
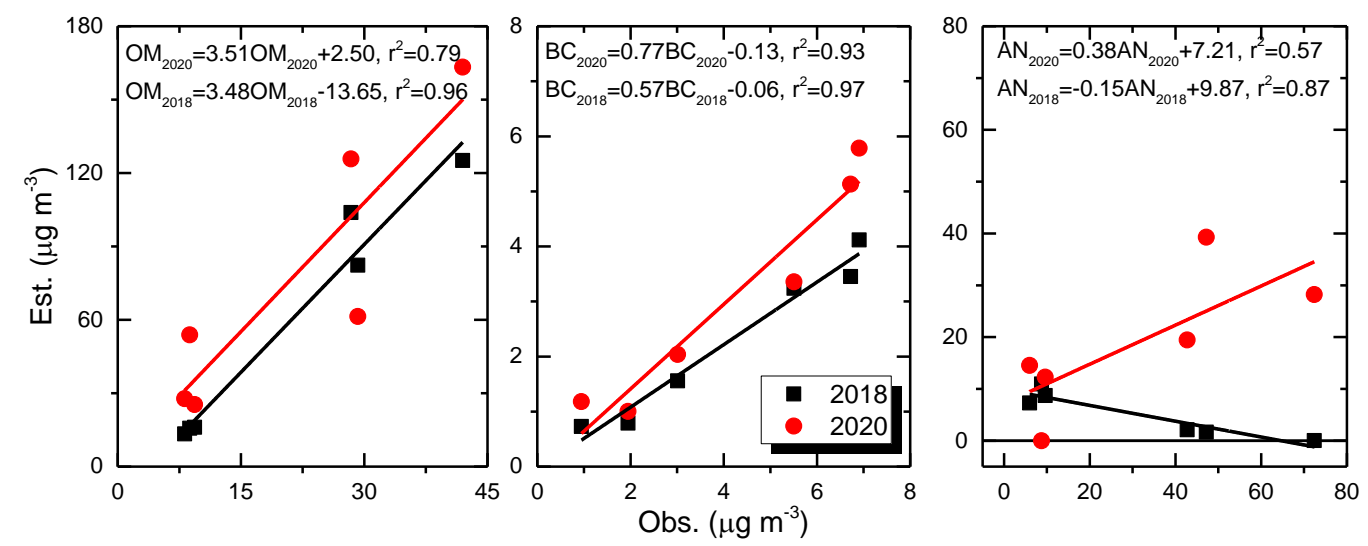

Figure S1. The comparison of aerosol components (OM, BC and AN) between this study and Zhang et al., 2018.

The daily volume fraction of $\mathrm{AW}_{\mathrm{f}}$ from the algorithm of 2020 and 2018 is present in figure $\mathrm{S} 2$. The 
volume fraction of $\mathrm{AW}_{\mathrm{f}}$ obtained by the two algorithms is consistent with the change of relative humidity. $\mathrm{AW}_{\mathrm{f}}$ from the algorithm of 2020 is slightly higher than that of 2018 . The new algorithm increases the low $\mathrm{AW}_{\mathrm{f}}$ when the $\mathrm{RH}$ is more than $40 \%$, obtaining more reasonable results.

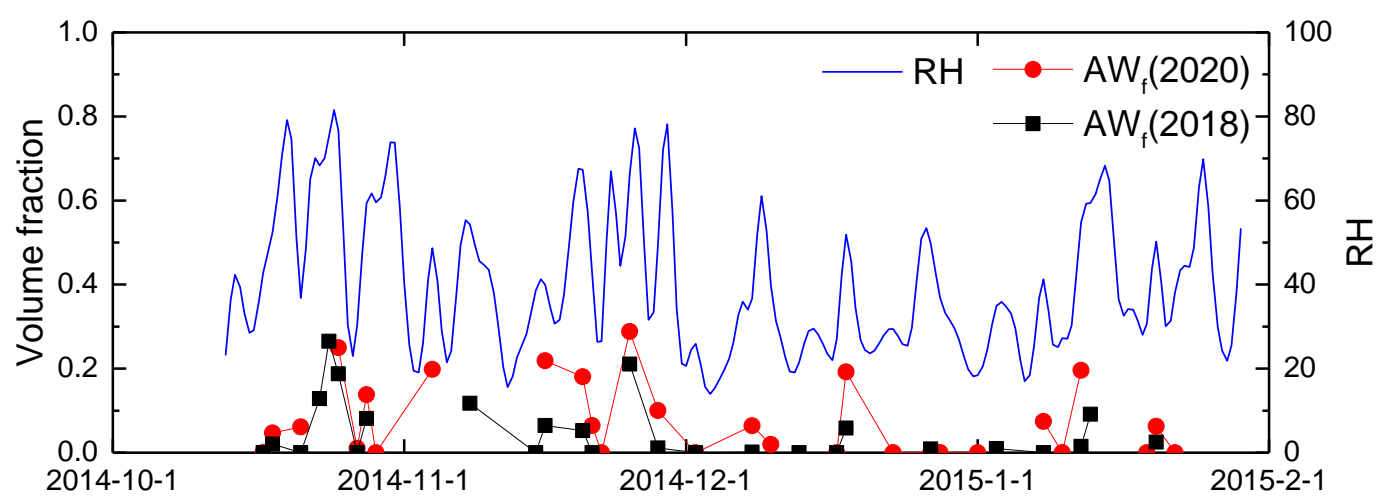

Figure S2. The RH and daily volume fraction of $\mathrm{AW}_{\mathrm{f}}$ from the algorithm of 2020 and 2018. 